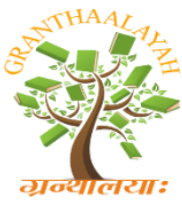

INTERNATIONAL JOURNAL OF RESEARCH GRANTHAALAYAH A knowledge Repository

Social

\title{
A STUDY OF PARTS OF SPEECH USED IN SELECTED NEWS ONLINE: A CASE STUDY OF SELECTED NEWS FROM BANGKOK POST ONLINE
}

\author{
Teerada Udomsup *1, Nutprapha K. Dennis ${ }^{2}$ \\ ${ }^{*} 1,2$ English Department, Graduate School, Ubon Ratchathani Rajabhat University, Thailand
}

DOI: https://doi.org/10.29121/granthaalayah.v5.i3.2017.1769

\begin{abstract}
The purpose of this study was to analyze parts of speech used from selected news online entitled "Thailand's anti-smoking YouTube hit". This study analyzed only words existed in selected news online from Bangkok Post, a Thai newspaper online. It focused on parts of speech used; noun, verb, adjective, adverb, pronoun, preposition, determination, conjunction and number. The researchers use only a website "parts-of-speech.info" to analyze and collect data. Selected news from Bangkok Post online entitled "Thailand's anti-smoking YouTube hit" was main material for this independent study. Parts-of-speech.info are a website used to collect and analyze data from the selected news. Parts-of-speech.info were used to analyze each sentence from the selected news. The results showed total of; adjective, adverb, conjunction, determiner, noun, number, preposition, pronoun and verb that existed in each sentence. The findings may be useful for English teachers who would like to teach all skills using online news as their teaching resources.
\end{abstract}

Keywords: Parts of Speech; Part-of-Speech Tagger; Online News.

Cite This Article: Teerada Udomsup, and Nutprapha K. Dennis. (2017). “A STUDY OF PARTS OF SPEECH USED IN SELECTED NEWS ONLINE: A CASE STUDY OF SELECTED NEWS FROM BANGKOK POST ONLINE." International Journal of Research - Granthaalayah, 5(3), 198-208. https://doi.org/10.29121/granthaalayah.v5.i3.2017.1769.

\section{Introduction}

Year after year English has been studied in Thailand, but to be successful in English is actually very complex. Certain skills must be mastered if someone wants to achieve a high level of proficiency in English. There are four skills in English which must be mastered: these are listening, speaking, reading and writing. The learner can enhance their speaking skills through conversation, debate, speech-making, role-playing and re-telling stories. Learners can write short stories, diaries and tell about unforgettable experiences to increase their writing skills. In order to increase reading skills, the students should read graded reading texts. Students must understand 
the meaning of each word in context in a sentence to increase their ability in the English language.

In present global education, online English websites enable students to assimilate many aspects of the English language, especially everyday English. Learning English through online English websites also assists one to practice English by listening, speaking, reading and writing. Cathy (1997), explain that the website is important to organizations and is recognized by all industries including educational organizations and institutions. The main benefit of English learning websites is that millions of students can be reached. Research has shown that total Internet user growth in Asia has increased more than 40\% from 2000 to 2008 (Internet World States, 2008).

Bangkok Post Online Website is one of the main media that helps Thai students to understand news and information, because it has various practices, activities and levels for the students, which they can choose to study, to improve the use of the English language. The learner can also study many different kinds of words, for example nouns, verbs, adjectives, and adverbs. The learner can analyze the function and kind of the word that is in the text. Learners often consider that it is a simple thing and it is not important to the learner. As English teacher, some lesson would look at the meaning of the text and ignore or neglect the function and kind of the words. Whereas, kinds of word are important components in English learning because those are very useful for us when we will express our utterances or write English sentences. One simple example is the difference between common nouns and proper nouns. Some simply stated, a proper noun is capitalized, whereas a common noun is not. Any grammar teacher, however, will tell students that the difference goes deeper than that: there are special rules for identifying and creating proper nouns, and these might take special studying to understand them completely, that students are still confused to about the type of compound words and part of speech.

The data sources for this study were taken from articles on online newspaper website "Bangkok Post" (www.bangkokpost.com) which covers topics from all around the world. This data was chosen because those articles contain many English nouns, but this site also increases our knowledge of what is happening now. Online newspapers is really popular nowadays, because the updating information is really quick. The information or the updating news will be posted on the site just in a few moments.

\section{Purposes of the study}

The purpose of this study was to analyze parts of speech used from selected news online entitled "Thailand's anti-smoking YouTube hit".

\section{Scope of the study}

This study analyzed only words existed in selected news online from Bangkok Post, a Thai newspaper online. It focused on parts of speech used; noun, verb, adjective, adverb, pronoun, preposition, determination, conjunction and number. The researcher use only a website "parts-ofspeech.info" to analyze and collect data.

\section{Significance of the Study}

The finding from this study will be beneficial as follows: 
- The findings may be useful for students who would like to learn English by themselves using online news because there are many activities to learn such as interactive \& printable English lessons in seven levels and all skills to learn included.

- The findings may be useful for English teachers who would like to teach all skills using online news as their teaching resources.

- This finding might be beneficial for interested people or other language learners, educational institutions and practitioners to develop the use of the English language. Moreover, it is also guideline language policy maker for curriculum design.

- Definition of Key Terms

- "Parts of Speech" in this Independent Study refers to words categorized into 9 categories; adjective, adverb, conjunction, determiner, noun, number, preposition, pronoun and verb.

- Parts-of-speech.info refers to a website used in this Independent Study to analyze and collect the data. It's a Parts-of-speech Tagging program.

- "Bangkok Post Online" refers to a Thai newspaper which published online along with hard-copy version daily.

- "News Online" in this Independent Study refers to published news on Bangkok Post online website.

\section{Parts-of-speech.info}

In corpus linguistics, part-of-speech tagging (POS tagging or POST), also called grammatical tagging or word-category disambiguation, is the process of marking up a word in a text (corpus) as corresponding to a particular part of speech, based on both its definition and its context-i.e., its relationship with adjacent and related words in a phrase, sentence, or paragraph. A simplified form of this is commonly taught to school-age children, in the identification of words as nouns, verbs, adjectives, adverbs, etc. (Wikipedia, n.d.).

According to Potiantong (2010) study, the result showed that the aims of the study the genre move structure and lexicon-grammatical features of main dish recipes. Forty recipes are selected and analyzed from one English cookbook. The result of the study shows that; noun is the mostly part of speech used in recipe writing (44.018\%) while pronoun is the least part of speech found from the study $(0.355 \%)$. The move structures of the recipes are: Introduction, Recipe Name, Relate Information, Ingredients Part, Cooking Instruction, Additional guidance, and Side Dish Recipe. In conclusion, the recipe writing is unique unlike other type of writing. The style of recipe writing is shaped by the structure that each part separated and can be seen clearly. The imperative sentences are popular used in this kind of writing more than other kind of sentence.

Surin (2005) studied English vocabularies of grade 6, grade 9 and grade 12 students 2008. The purposes of this research were to survey English vocabularies of grade 6, grade 9 and grade 12 students, to classify these vocabularies in two alphabetical A-Z groups, and to specify their parts of speech and word classes. For their parts of speech, it was found that most of grade 6 students' vocabularies i.e., 1,343 words $(63.98 \%)$ were nouns, while the least of them i.e., 11 words $(0.52 \%)$ were pronouns. For grade 9 students' vocabularies, most of them i.e., 1,763 words $(55.21 \%)$ were also nouns, but the least of them i.e., 5 words $(0.16 \%)$ were interjections. Moreover, most of grade 12 students' vocabularies i.e., 1,674 words (50.51\%) were nouns, but the least of them i.e., one word $(0.03 \%)$ was a pronoun. 
The purpose of the article written by Chumchai (2008) is to analyze the types of adjectives and adjective functions in a noun phrase found in an online news site "The Jakarta Globe" Online news are very well known in all circles, both among young people and adults, because the news were very easy to access. The information and latest news provided by the online news website can be published in a very quick time, without waiting for a longer period of time, just a few minutes were enough to gain all information for the interested users. This study uses the qualitative method, as well as the best possible literature method. The theory which is used as a reference in this research is a theory about types of adjectives by AJ Thomson and A.V. Martinet in his book with the title: "A Practical English Grammar Use", plus the theory of functions of word properties by Qurik in his book with the title: A Comprehensive Grammar of the English Language. The results of this study indicate that there are six types of adjectives in the British language: demonstrative adjectives, distributive adjectives, quantitative adjectives, interrogative adjectives, possessive adjectives, and adjectives of quality, as well as three function words properties in a noun phrase: attributive functions, postpositive functions, and adjectives as "the head of the noun phrase".

\section{Materials and Methods}

\section{Setting}

The data of this independent study was based on one selected news from Bangkok Post online.

\section{Materials}

Selected news from Bangkok Post online entitled "Thailand's anti-smoking YouTube hit" was main material for this independent study. This is the link for the material used http://www.bangkokpost.com/learning/learning-news/300929/thailand-anti-smoking-youtube-hit Parts-of-speech.info is a website used to collect and analyze data from the selected news. The core of Parts-of-speech.info is based on the Stanford University Part-Of-Speech-Tagger.

\section{Data Collection and Analysis}

Parts-of-speech.info were used to analyze each sentence from the selected news. The results showed total of; adjective, adverb, conjunction, determiner, noun, number, preposition, pronoun and verb that existed in each sentence.

\section{Results and Discussions}

This section presents results of the study and discussion after the result reveal. This study used a program from "Part-of-speech.info" to parse in the selected news. The program use color to show type of words as showed below. 


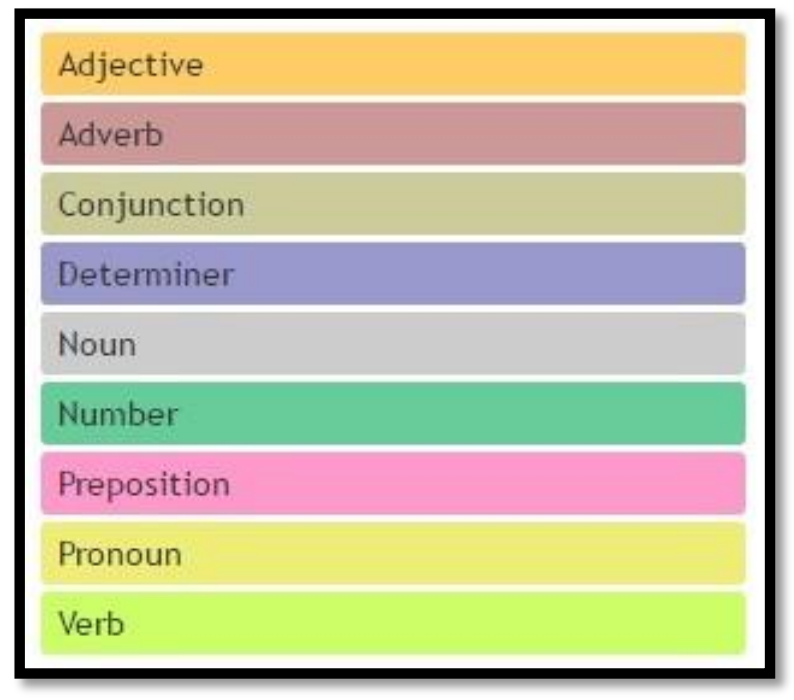

Figure 1: Identified colors to analyze parts of speech

\section{Word Analysis Section 1}

Thailand's anti-smoking YouTube hit

The news topic has been analyzed by the program and the results were; Thailand ( $n$. $) /$ antismoking (adj.) / YouTube (n.) / hit (n.)

There were 3 nouns with one adjective used in the news topic.

\section{Word Analysis Section 2}

A Thai anti-smoking advert goes viral on Youtube with over two million views in two weeks and winning praise from around the world.

The sentence has been analyzed by the program and the results were; A (det.) Thai (n.) antismoking (adj.) advert (n.) goes (v.) viral (adj.) on (prep.) Youtube (n.) with (prep.) over (prep.) two (num.) million (num.) views (n.) in (prep.) two(num.) weeks (n.) and (conj.) winning (v.) praise (n.) from (prep.) around (prep.) the (det.) world (noun).

According to the results, the word "winning" showed as "verb" analyzed by the program. However, the result was a mistake by the program analyzed because "winning" was modified the word "praise" as an "adjective".

\section{Word Analysis Section 3}

Message hits home

Thai anti-smoking video campaign making waves on YouTube (Yanapon Musiket). The phrases have been analyzed by the program and the results were; Message (n.) hits (v.) home (n.) <phrase as news topic> / Thai (n.) anti-smoking (adj.) video (n.) campaign (n.) making (v.) waves(n.) on (prep.) YouTube (n.) -Yanapon (n.) Musiket (n.)

According to the results, there're some points need to be discussed. There were 2 phrases in this analyzed section. First phrase, "Message hits home", was a sub-topic of the news, and the second one, "Thai anti-smoking video campaign making waves on YouTube". In this second phrase, the program showed that "Thai" is a noun as a word itself, but in this phrase Thai modified "campaign" as an adjective. Moreover, the word "video" and " anti-smoking" are also adjectives modifying the word "campaign". The word "making" was not a verb in this phrase. There was 
no "verb" in this phrase. The word "Yanapon" and "Musiket" showed as nouns, but it's a proper noun for a person name "Yanapon Musiket".

\section{Word Analysis Section 4}

The camera follows a little boy and a girl, walking up to men and women smoking in a public area outside an office building. Cigarette in hand, and in the casual manner of one smoker to another, they ask the adults for a lighter.

The sentences have been analyzed by the program and the results were; The (det.) camera ( $n$.) follows (v.) a (det.) little (adj.) boy (n.) and (conj.) a (det.) girl (n.), walking (v.) up (-) to (-) men (n.) and (conj.) women (n.) smoking (n.) in (prep.) a (det.) public (adj.) area (n.) outside (prep.) an (det.) office (n.) building (n.). Cigarette (n.) in (prep.) hand (n.), and (conj.) in (prep.) the (det.) casual (adj.) manner (n.) of (prep.) one (num.) smoker (n.) to (-) another (det.), they (pron.) ask (v.) the (det.) adults (n.) for (prep.) a (det.) lighter (adj.).

According to the results, the word "walking" was identified as verb, and "up" couldn't identify by the program. Based on the previous studies, "walking up" should be identified as a verb, a two-word verb. The words "office" and "building" identified as nouns, however, "office" modified "building", so that "office" was an adjective, not noun in this sentence. The word "lighter" in this sentence was a noun, not adjective, means "a mechanical device used in lighting cigarettes, cigars, or pipes for smoking." (www.dictionary.com)

\section{Word Analysis Section 5}

Shocked by the request, the smoking adults refuse to lend a light and begin lecturing the kids about the harm of tobacco.

The sentence has been analyzed by the program and the results were; Shocked (v.) by (prep.) the (det.) request (n.), the (det.) smoking (n.) adults (n.) refuse (v.) to (-) lend (v.) a (det.) light (n.) and (conj.) begin (v.) lecturing (v.) the (det.) kids (n.) about (prep.) the (det.) harm (n.) of (prep.) tobacco (n.).

According to results, the word "smoking" was an adjective modifying "adult", not a noun. The word "lecturing" was a noun, not a verb in this sentence.

\section{Word Analysis Section 6}

Tension ensues and things get emotional and the kids shoot back: "If smoking is bad, why do you smoke?" They pass on a piece of paper to the smokers before walking away. The sentences have been analyzed by the program and the results were; Tension (n.) ensues (n.) and (conj.) things (n.) get (v.) emotional (adj.) and (conj.) the (det.) kids (n.) shoot (v.) back (adv.): "If (prep.) smoking (n.) is (v.) bad (adj.), why (adv.) do (v.) you (pron.) smoke (v.)?" They (pron.) pass (v.) on (prep.) a (det.) piece (n.) of (prep.) paper (n.) to (-) the (det.) smokers (n.) before (prep.) walking (v.) away (adv.).

\section{Word Analysis Section 7}

The note reads: "You're worried about me but why not about yourself?" The sentence has been analyzed by the program and the results were; The (det.) note (n.) reads (v.): "You (pron.) 're (v.) worried (v.) about (prep.) me (pron.) but (conj.) why (adv.) not (adv.) about (prep.) yourself (pron.)?" 


\section{Word Analysis Section 8}

This is a Thai anti - tobacco campaign titled Smoking Kids, and its creepy, urgent power comes from its reality-TV style - a hand-held camera shot from various angles that seem to catch those smoking people unawares, plus the prankish twist that delivers the message and makes the adults blush.

The sentence has been analyzed by the program and the results were; This (det.) is (v.) a (det.) Thai (n.) anti-tobacco (adj.) campaign (n.) titled (v.) Smoking (n.) Kids (n.), and (conj.) its (pron.) creepy (adj.), urgent (adj.) power (n.) comes (v.) from (prep.) its (pron.) reality-TV (adj.) style (n.) - a (det.) hand-held (adj.) camera (n.) shot (n.) from (prep.) various (adj.) angles (n.) that (det.) seem (v.) to (-) catch (v.) those (det.) smoking (n.) people (n.) unawares (adv.), plus (conj.) the (det.) prankish (adj.) twist (n.) that (det.) delivers (v.) the (det.) message (n.) and (conj.) makes (v.) the (det.) adults (n.) blush (v.).

\section{Word Analysis Section 9}

Posted on YouTube, the video recently went viral. After just two weeks, the number of viewers has reached two million. Comments have flooded in from all over the world, mostly praising the effectiveness and intelligence of the campaign.

The sentences have been analyzed by the program and the results were; Posted (v.) on (prep.)YouTube (n.), the (det.) video (n.) recently (adv.) went (v.) viral (adj.). After (prep.) just (adv.) two (num.) weeks (n.), the (det.) number (n.) of (prep.) viewers (n.) has (v.) reached (v.) two (num.) million (num.). Comments (n.) have (v.) flooded (v.) in (prep.) from (prep.) all (det.) over (prep.) the (det.) world (n.), mostly (adv.) praising (v.) the (det.) effectiveness (n.) and (conj.) intelligence (n.) of (prep.) the (det.) campaign (n.).

\section{Word Analysis Section 10}

The Thai Health Promotion Foundation commissioned Ogilvy \& Mather to create the video. It is a campaign made specifically for social media websites and not for television, and the first in a series of anti-smoking videos the national health agency is planning to roll out.

The sentences have been analyzed by the program and the results were; The (det.)Thai (n.) Health (n.) Promotion (n.) Foundation (n.) commissioned (v.) Ogilvy (n.) \& (conj.) Mather (n.) to (-) create (v.) the (det.) video (n.). It (pron.) is (v.) a (det.) campaign (n.) made (v.) specifically (adv.) for (prep.) social (adj.) media (n.) websites (n.) and (conj.) not (adv.) for (prep.) television (n.), and (conj.) the (det.) first (adj.) in (prep.) a (det.) series (n.) of (prep.) anti-smoking (adj.) videos (n.) the (det.) national (adj.) health (n.) agency (n.) is (v.) planning (v.) to (-) roll (v.) out $(-)$.

\section{Word Analysis Section 11}

"We have seen a number of anti-smoking campaigns, local and international," says Nopadol Srikieatikajohn, executive creative director of Ogilvy \& Mather Bangkok. The sentence has been analyzed by the program and the results were; "We (pron.) have (v.) seen (v.) a (det.) number (n.) of (prep.) anti-smoking (adj.) campaigns (n.), local (adj.)) and (conj.) international (adj.)," says (v.) Nopadol (n.) Srikieatikajohn (n.), executive (adj.) creative (adj.) director (n.) of (prep.) Ogilvy (n.) \& (conj.) Mather (n.) Bangkok (n.). 


\section{Word Analysis Section 12}

"For this one, we decided to make it real and actionable. It is a simple production but we focus more on ideas and copywriting in order to communicate with the audience directly and effectively."

The sentences have been analyzed by the program and the results were; "For (prep.) this (det.) one (num.), we (pron.) decided (v.) to (-) make (v.) it (pron.) real (adj.) and (conj.) actionable (adj.). It (pron.) is (v.) a (det.) simple (adj.) production (n.) but (conj.) we (pron.) focus (v.) more (conj.) on (prep.) ideas (n.) and (conj.) copywriting (n.) in (prep.) order (n.) to (-) communicate (v.) with (prep.) the (det.) audience (n.) directly (adv.) and (conj.) effectively (adv.)."

\section{Word Analysis Section 13}

At the end of the video, a message informs us that almost every adult who receives the antismoking note passed on by the children instantly stops to think, then throws the cigarette away. The sentence has been analyzed by the program and the results were; At (prep.) the (det.) end (n.) of (prep.) the (det.) video (n.), a (det.) message (n.) informs (v.) us (pron.) that (prep.) almost (adv.) every (det.) adult (n.) who (pron.) receives (v.) the (det.) anti-smoking (adv.) note (n.) passed (v.) on (-) by (prep.) the (det.) children (n.) instantly (adv.) stops (v.) to (-) think (v.), then $(a d v$.$) throws (v.) the (det.) cigarette (n.) away (adv.).$

\section{Word Analysis Section 14}

None of them throws away the note. And most importantly, the message tells us that the number of phone inquiries by smokers who wanted to quit has increased by $40 \%$. The sentences have been analyzed by the program and the results were; None (n.) of (prep.) them (pron.) throws (v.) away (-) the (det.) note (n.). And (conj.) most (adv.) importantly (adv.), the (det.) message (n.) tells (v.) us (pron.) that (prep.) the (det.) number (n.) of (prep.) phone (n.) inquiries (n.) by (prep.) smokers (n.) who (pron.) wanted (v.) to (-) quit (v.) has (v.) increased (v.) by (prep.) 40 (num.) \% (n.).

\section{Word Analysis Section 15}

Supatnuj Sorndamrih, social marketing specialist at the foundation, says the agency is pleased with the instant success of the campaign.

The sentence has been analyzed by the program and the results were; Supatnuj (n.) Sorndamrih (n.), social (adj.) marketing (n.) specialist (n.) at (prep.) the (det.) foundation (n.), says (v.) the (det.) agency (n.) is (v.) pleased (v.) with (prep.) the (det.) instant (adj.) success (n.) of (prep.) the (det.) campaign (n.).

\section{Word Analysis Section 16}

"The video really helps us get this message across."

The sentence has been analyzed by the program and the results were; "The (det.) video (n.) really (adv.) helps (v.) us (pron.) get (v.) this (det.) message (n.) across (prep.)."

\section{Word Analysis Section 17}

Supatnuj said the video was based on research and the foundation believes that smokers know the danger of tobacco well but choose to ignore it.

The sentences have been analyzed by the program and the results were; Supatnuj (n.) said (v.) the (det.) video (n.) was (v.) based (v.) on (prep.) research (n.) and (conj.) the (det.) foundation 
$(n$.$) believes (v.) that (prep.) smokers (n.) know (v.) the (det.) danger (n.) of (prep.) tobacco (n.)$ well (adv.) but (conj.) choose (v.) to (-) ignore (v.) it (pron.).

\section{Discussion}

According to the results above, the website Parts-of-speech.info was the main instrument helped the researcher to analyze and collect the data. The Parts-of-speech.info website is Parts-of-speech Tagging program. Part-of-speech tagging (Wikipedia, n.d.) is harder than just having a list of words and their parts of speech, because some words can represent more than one part of speech at different times, and because some parts of speech are complex or unspoken. This is not rarein natural languages (as opposed to many artificial languages), a large percentage of word-forms are ambiguous. For example, even "dogs", which is usually thought of as just a plural noun, can also be a verb: "The sailor dogs the hatch." Correct grammatical tagging will reflect that "dogs" is here used as a verb, not as the more common plural noun. Grammatical context is one way to determine this; semantic analysis can also be used to infer that "sailor" and "hatch" implicate "dogs" as 1) in the nautical context and 2) an action applied to the object "hatch" (in this context, "dogs" is a nautical term meaning "fastens (a watertight door) securely").

According to the results, the word "walking" was identified as verb, and "up" couldn't identify by the program. Based on the previous studies, "walking up" should be identified as a verb, a two-word verb. The words "office" and "building" identified as nouns, however, "office" modified "building", so that "office" was an adjective, not noun in this sentence. The word "lighter" in this sentence was a noun, not adjective, means "a mechanical device used in lighting cigarettes, cigars, or pipes for smoking." (www.dictionary.com)

Most parts of speech can be divided into sub-classes. Prepositions can be divided into prepositions of time, prepositions of place etc. Nouns can be divided into proper nouns, common nouns, concrete nouns etc. It is important to know that a word can sometimes be in more than one part of speech. For example with the word "increase"; "Increase" can be a "verb" e.g. "Prices increased." And "increase" can also be a "noun" e.g. "There was an increase in the number of followers" (Parts of Speech, n.d.).

\section{Conclusions \& Recommendations}

The purpose of this study was to analyze parts of speech used from selected news online entitled "Thailand's anti-smoking YouTube hit". This study analyzed only words existed in selected news online from Bangkok Post, a Thai newspaper online. It focused on parts of speech used; noun, verb, adjective, adverb, pronoun, preposition, determination, conjunction and number. The researcher uses only a website "parts-of-speech.info" to analyze and collect data. Selected news from Bangkok Post online entitled "Thailand's anti-smoking YouTube hit" was main material for this independent study. This is the link for the material used http://www.bangkokpost.com/learning/learning-news/300929/thailand-anti-smoking-youtube-hit (See Appendix).

Parts-of-speech.info is a website used to collect and analyze data from the selected news. The core of Parts-of-speech.info is based on the Stanford University Part-Of-Speech-Tagger. Parts- 
of-speech.info were used to analyze each sentence from the selected news. The results showed total of; adjective, adverb, conjunction, determiner, noun, number, preposition, pronoun and verb that existed in each sentence. According to the results above, the website Parts-of-speech.info was the main instrument helped the researcher to analyze and collect the data. The Parts-ofspeech.info website is Parts-of-speech Tagging program. Most parts of speech can be divided into sub-classes. Prepositions can be divided into prepositions of time, prepositions of place etc. Nouns can be divided into proper nouns, common nouns, concrete nouns etc. It is important to know that a word can sometimes be in more than one part of speech. For example with the word "increase"; "Increase" can be a "verb" e.g. "Prices increased." And "increase" can also be a "noun" e.g. "There was an increase in the number of followers" (Parts of Speech, n.d.). Example of the finding showed below. According to the results, the word "walking" was identified as verb, and "up" couldn't identify by the program. Based on the previous studies, "walking up" should be identified as a verb, a two-word verb. The words "office" and "building" identified as nouns, however, "office" modified "building", so that "office" was an adjective, not noun in this sentence. The word "lighter" in this sentence was a noun, not adjective, means "a mechanical device used in lighting cigarettes, cigars, or pipes for smoking.” (www.dictionary.com)

The finding from this study will be beneficial as follows:

The findings may be useful for students who would like to learn English by themselves using online news because there are many activities to learn such as interactive \& printable English lessons in seven levels and all skills to learn included. The findings may be useful for English teachers who would like to teach all skills using online news as their teaching resources. This finding might be beneficial for interested people or other language learners, educational institutions and practitioners to develop the use of the English language. Moreover, it is also guideline language policy maker for curriculum design.

\section{Recommendation}

- Students who study English can utilize this analysis as a useful resource to increase their understanding of grammatical words such as sentences, tense, etc., at online English news more easily and more efficient.

- Students who study English can utilize this analysis as a useful source to increase their understanding of parts of speech used in other online news providers, enabling them to read online English news more easily without the pressure having a teacher behind him or her.

- English instructors, teachers, parents and guardians who would like to teach sentence structures by using online news can easily use this case study as their teaching resource to enhance learners' educational outcome intensively.

- This study can truly affect the learners to learn the English language by using online news website. It's certain that this study can provide basic knowledge for those who are interested in studying linguistic elements in any other forms, further more studies should analyze the grammatical structures used in English news online as well.

\section{Acknowledgements}

This independent study would not have been completed without the generous help from many people. First of all, I would like to express my deepest gratitude and appreciation to my advisor, for 
her valuable suggestions and creative criticism throughout the course of the study. I must also express my very profound gratitude to my parents and all lovely friends for providing me with unfailing support and continuous encouragement throughout my years of study and through the process of researching and writing this independent study. This accomplishment would not have been possible without them. Thank you.

\section{References}

[1] Adjective. (Online). Retrieved November 6, 2014 from partsofspeech.org

[2] Adverb. (Online). Retrieved November 6, 2014 from partsofspeech.org

[3] Benson, M. (1985). Collocations and Idioms. Dictionaries, Lexicography and Language Learning. Oxford University Press.

[4] Cathy, M. (1990). Vocabulary. Oxford, Oxford University Press.

[5] Chumchai, C. (2008). An Analysis of Linguistics Features in Selected Korean Movie Synopses. Master's Thesis. Chiangrai Rajabhat University.

[6] Conjunction. (Online). Retrieved November 6, 2014 from partsofspeech.org

[7] Determiner. (Online). Retrieved November 6, 2014 from partsofspeech.org

[8] Internet World States. (2008). Asia Internet Usage States and Populations Statistics. Retrieved June 8, 2014 from http://www.internetworldstats.com/stats3.htm.

[9] Nouns. (Online). Retrieved November 6, 2014 from partsofspeech.org

[10] Parts of Speech. (n.d.). English Grammar. Retrieved August 4, 2015 from http://www.grammar.cl/english/parts-of-speech.htm

[11] Part-of-speech tagging. (n.d.). In Wikipedia. Retrieved January 20, 2016, from https://en.wikipedia.org/wiki/Part-of-speech_tagging.

[12] Phanphanich, P. (1999). An Analysis of English Usage in Political News in English Language Newspaper. Master of Arts Independent Study. Ubon Ratchathani Rajabhat University.

[13] Preposition. (Online). Retrieved November 6, 2014 from partsofspeech.org

[14] Potiantong, K. (2010). A Genre Analysis of the Main Dish Recipe. Master of Arts Independent Study. Ubon Ratchathani Rajabhat University.

[15] Pronoun. (Online). Retrieved November 6, 2014 from partsofspeech.org

[16] Seasiaw, D. (2005). The Genre of Motoring News in the Nation and the Bangkok Post: A Case Study. Master of Arts Independent Study. King Mongkut's Institute of Technology North Bangkok.

[17] [17] Surin, B. The Analysis of English Usage in Cosmetic Advertisement Headlines. Master of Arts Independent Study. King Mongkut's Institute of Technology North Bangkok.

[18] Suvaree, P. (2006). The Study of Language Used in Airlines' Advertisements. Master of Arts Independent Study. King Mongkut's Institute of Technology North Bangkok.

[19] Tadena, C. I. (2014). Parts of Speech. (Online). Available from http://partsofspeech.org.

[20] Verb. (Online). Retrieved November 6, 2014 from partsofspeech.org

[21] William, J. D. (2005). Teacher's Grammar Book. Lawrence Erlbaum Associates.

*Corresponding author.

E-mail address: nootprapa@gmail.com 